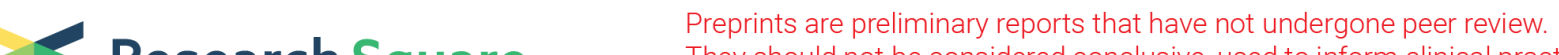 Research Square They should not be considered conclusive, used to inform clinical practice, or referenced by the media as validated information.
}

\section{Hyponatremia and hypophysitis induced by ipilimumab and nivolumab in patients with lung squamous cell carcinoma: a case report}

\section{Lingjie Hou}

Harbin Medical University Cancer Hospital

Hong Zhang

Harbin Medical University Cancer Hospital

\section{Yanan Shao}

Harbin Medical University Cancer Hospital

Yang Zhou

Harbin Medical University Cancer Hospital

Hongquan Zhang

Peking University Health Science Centre

Meng Wang ( $\nabla$ wangmeng@hrbmu.edu.cn )

Harbin Medical University Cancer Hospital

\section{Case Report}

Keywords: Lung squamous cell carcinoma, Nivolumab, Ipilimumab, Hyponatremia, Hypophysitis

Posted Date: February 22nd, 2022

DOI: https://doi.org/10.21203/rs.3.rs-1374856/v1

License: () (1) This work is licensed under a Creative Commons Attribution 4.0 International License.

Read Full License 


\section{Abstract}

Background: Immune checkpoint inhibitors (ICls) have transformed the prognosis of patients with advanced lung squamous cell carcinoma (SCC). As the use of checkpoint inhibitors increases in a variety of malignancies, immune-related adverse effects will become more common.

Case report: Here, we present a case of a 64-year-old man with advanced stage lung SCC who developed hyponatremia and hypophysitis after treatment with ipilimumab and nivolumab. The patient underwent effective treatment with steroids, hyperosmolar therapy, and fluid restriction.

Conclusion: This case highlights the potentially life-threatening complications of ICls, and it shows that early recognition and complete evaluation are critical in clinical practice.

\section{Introduction}

Lung cancer is the leading cause of cancer deaths in China and worldwide [1]. In patients who have advanced non-small-cell lung cancer (NSCLC) without oncogenic driver mutations, first-line treatment is platinum-based combination chemotherapy. This treatment has been the standard of care for the past three decades with few improvements, and it is characterised by moderate-to-severe toxicities, including haematological adverse events and non-haematological toxicities, such as nausea, vomiting, diarrhea, and alopecia. However, in the last decade, immune checkpoint inhibitors (ICIs), specifically programmed cell death 1 (PD-1) pathway inhibitors, have changed the treatment paradigm of advanced NSCLC.

In recent years, ICls have become the standard of care in the treatment of advanced NSCLC, either in the first-line therapy (monotherapy in selected patients or in combination with chemotherapy) or beyond [2, 3]. In a broad range of cancers, nivolumab (a fully human IgG4-PD-1 ICl antibody) showed durable responses and encouraging survival as monotherapy or in combination with ipilimumab (a fully human IgG1 cytotoxic T lymphocyte antigen-4 (CTLA-4) ICl antibody) $[4,5,6,7]$.

Combination therapy with nivolumab and ipilimumab has been studied in patients with NSCLC, and it showed an encouraging result in the first-line settings [8]. However, the use of checkpoint inhibitors in cancer therapy is complicated by a unique spectrum of immune-related adverse events (irAEs). IrAEs include, but are not limited to, colitis, thyroiditis, hypophysitis, arthritis, dermatitis, and primary adrenal insufficiency [9]. In this report, we describe a patient who developed hyponatremia and hypophysitis after treatment with ipilimumab and nivolumab for NSCLC.

\section{Case Report}

A 64-year-old male, who had a 20-year history of smoking, experienced cough chest pain and the presence of bloody sputum for half a month. Chest computed tomography (CT) showed a 4.3-cm irregular mass in the left upper lobe with mediastinal lymph node metastasis and right interlobular pleural metastasis in August 2018. Brain magnetic resonance imaging (MRI) did not show brain metastasis. 
Findings on bronchoscopy and biopsy confirmed a diagnosis of squamous cell carcinoma. Immunohistochemistry (IHC) staining revealed that the expression was positive for $\mathrm{CK} 5 / 6, \mathrm{P} 63$, and $\mathrm{P} 40$, and it was negative for TTF-1 and Napsin A. He was diagnosed with clinical stage IV (cT2N2M1) squamous cell carcinoma of the lung.

The serum levels of carcinoembryonic antigen (CEA) and squamous cell carcinoma antigen (SCC) were increased to $47.19 \mathrm{ng} / \mathrm{mL}$ (reference range, $0-5 \mathrm{ng} / \mathrm{mL}$ ) and $3.2 \mathrm{ng} / \mathrm{mL}$ (reference range, $0-1.5 \mathrm{ng} / \mathrm{mL}$ ), respectively. The specimen showed no epidermal growth factor receptor (EGFR) mutations and no anaplastic lymphoma kinase (ALK) translocations. Therefore, we tested the PD-L1 status, and IHC staining showed that over $50 \%$ of the tumor cells had membranous staining of PD-L1 (Fig. 1). Dako (Monoclonal Mouse Anti-PD-L1, Clone 22C3 antibody) was used for PD-L1 immunostaining.

The patient had received ICls therapy consisting of nivolumab ( $3 \mathrm{mg} / \mathrm{kg}$ every 2 weeks) and ipilimumab ( $1 \mathrm{mg} / \mathrm{kg}$ every 6 weeks) as first-line therapy in August 16, 2018. At that time, his general condition corresponded to Eastern Cooperative Oncology Group performance status (ECOG-PS) 1. One month later, a CT scan revealed that the lesions in the lung decreased in size by more than $30 \%$, meeting the response evaluation criteria for a partial response (PR) (Fig. 2). Initial treatment was well tolerated, after the 13th cycle of nivolumab and 5th cycle of ipilimumab treatment, he visited our hospital complaining of diarrhea, nausea, vomiting, fatigue, and headache. During that period, his ECOG-PS score had also rapidly deteriorated to 2 .

On the day of admission, investigation revealed the following findings: serum sodium $119 \mathrm{mmol} / \mathrm{L}$ [(137-147) $\mathrm{mmol} / \mathrm{L}]$, chloride $84 \mathrm{mmol} / \mathrm{L}$ [(99-110) $\mathrm{mmol} / \mathrm{L}]$, TSH $33.66 \mathrm{ulU} / \mathrm{mL}$ [(0.27-4.2) ulU/mL], FT4 $10.89 \mathrm{pmol} / \mathrm{L}$ [(12-22) pmol/L], FT3 $3.12 \mathrm{pmol} / \mathrm{L}$ [(3.1-6.8) pmol/L], and creatine kinase (CK) 182 $\mathrm{U} / \mathrm{L}[(35-232) \mathrm{U} / \mathrm{L}]$. Additionally, there was a decrease in levels of $\mathrm{ACTH}<0.22 \mathrm{pmol} / \mathrm{L}[0.22-440 \mathrm{pmol} / \mathrm{L}]$ and cortisol $7.7 \mathrm{nmol} / \mathrm{L}$ [171-536 nmol/L]. No abnormality was found in the blood or urine routine test. Subsequently, MRI scan of the pituitary gland showed no space-occupying lesions, but it showed obvious enhancement in pituitary stalk indicative of hypophysitis (Fig. 3).

The patient was then treated with steroids, fluid restriction and hypertonic saline until resolution of the syndrome. During this period, the patient was commenced on oral steroids and $10 \%$ sodium chloride treatment, and steroid taper was prescribed at $60 \mathrm{mg}$ of prednisone daily to $10 \mathrm{mg}$ daily over the course of one month. It was later tapered off over a course of 2 weeks. After 15 days, the patient's symptoms resolved and serum sodium returned to normal. Then the patient stopped taking ipilimumab and continued nivolumab therapy without interruption.

At the last review, the patient had completed 25 cycles of nivolumab and was completely well with normal blood biochemistry (serum sodium $141 \mathrm{mmol} / \mathrm{L}$, ACTH 86 pmol/L) (Fig. 4A). In addition, changes in the thyroid function were observed throughout the treatment (Fig. 4B). Contrast-enhanced CT imaging revealed complete reduction of the mass in the lung with no other evidence of irAEs. Serum levels of CEA and SCC returned to normal levels after two months of treatment (Fig. 4C). 


\section{Discussion}

In this report, we present a patient who developed hyponatremia and hypophysitis after treatment with nivolumab and ipilimumab for NSCLC. ICls therapies have shown favorable outcomes in patients with lung cancer. However, these medicines also cause immune responses to some specific organs and cause irAEs, such as colitis, rash, and hepatitis $[10,11]$. ICls can also cause autoimmune disorders, such as endocrine dysfunction, including thyroid dysfunction and hypopituitarism, during the treatment period $[12,13,14]$, and these factors may limit the clinical application of these therapies.

The combination of nivolumab and ipilimumab increased the risk of irAEs in patients with advanced cancer [15]. Ipilimumab is a well-recognized cause of pituitary dysfunction (hypophysitis and/or hypopituitarism), and it is reported to occur in up to $25 \%$ of patients who often present with multiple anterior pituitary hormone deficiencies $[16,17]$. There is often an assumption in patients with malignancy that hyponatremia is most frequently due to hypopituitarism and syndrome of inappropriate anti-diuretic hormone (SIADH) $[18,19]$. The isolated ACTH deficiency secondary to checkpoint inhibitor-induced hypophysitis is also a well-recognized complication of ipilimumab. In this case report, MRI scan of the pituitary gland and decrease in the serum ACTH level can lead to the diagnosis of hypophysitis. The precise mechanism(s) of immune-mediated hypophysitis remains unclear; it is likely linked to the antiCTLA4 antibody binds to the CTLA4 antigen on the pituitary gland, resulting in the activation of the classical complement cascade and an inflammatory response [20].

In addition, combined therapy shows a striking $44 \%$ increase in the risk of diarrhea and immune-related colitis, which are closely associated with hyponatremia [21]. Hassel et al. reported that the frequency of diarrhea was $34 \%$ for ipilimumab, $17 \%$ for nivolumab, and $43 \%$ for combination therapy. The reported frequency of colitis was $12 \%$ for ipilimumab, $1 \%$ for nivolumab, and $14 \%$ for combination therapy [22]. Hyponatremia is the most common electrolyte disorder in hospitalized patients, and it most frequently occurs due to diarrhea and colitis.

This case illustrates the importance of recognizing potential severe adverse events of ICls. ICls-induced hyponatremia and hypophysitis are the possible side effects of the treatment. Evaluation of the pituitary gland with MRI in conjunction with the serum ACTH level may be helpful in detecting treatment-related hypophysitis and preventing life-threatening irAEs.

\section{Abbreviations}

ICls: immune checkpoint inhibitors

SCC: squamous cell carcinoma

NSCLC: non-small-cell lung cancer

PD-1: programmed cell death 1 
CTLA-4: cytotoxic T lymphocyte antigen-4

irAEs: immune-related adverse events

CT: computed tomography

MRI: magnetic resonance imaging

IHC: immunohistochemistry

CEA: carcinoembryonic antigen

EGFR: epidermal growth factor receptor

ALK: anaplastic lymphoma kinase

ECOG-PS: Eastern Cooperative Oncology Group performance status

PR: partial response

CK: creatine kinase

SIADH: syndrome of inappropriate anti-diuretic hormone

\section{Declarations}

\section{Availability of data and materials}

All generated or analyzed during this study are included in this article.

\section{Acknowledgments}

We thank LetPub (www.letpub.com) for its linguistic assistance during the preparation of this manuscript.

\section{Funding}

This work was supported by the Natural Science Foundation of Heilongjiang Province (No.LH2019H039); the Haiyan Foundation of Harbin Medical University Cancer Hospital (No.JJZD2020-09).

\section{Author information}

\section{Affiliations}

Department of Respiratory Medical Oncology, Harbin Medical University Cancer Hospital, Harbin, China Lingjie Hou, Hong Zhang, Yanan Shao, Meng Wang 
Department of Immunology, Tianjin Medical University Cancer Institute and Hospital, National Clinical Research Center for Cancer, Key Laboratory of Cancer Prevention and Therapy, Key Laboratory of Immunology and Biotherapy, Tianjin's Clinical Research Center for Cancer, Tianjin, China

Lingjie Hou

Department of Radiology, Harbin Medical University Cancer Hospital, Harbin, China

Yang Zhou

Department of Human Anatomy, Histology, and Embryology, Key Laboratory of Carcinogenesis and Translational Research (Ministry of Education) and State Key Laboratory of Natural and Biomimetic Drugs, Peking University Health Science Centre, Beijing, China

Hongquan Zhang

Contributions

All of the authors contributed to the conception of the study, analysis of the data, writing of the manuscript, and approval of the final draft.

Corresponding author

Correspondence to Meng Wang.

Ethics approval and consent to participate

The case report was approved by the Ethics Committee of Harbin Medical University. The clinical information presented in this case report was obtained from the medical records of Harbin Medical University Cancer Hospital.

Consent for publication

Written informed consent was obtained from the patient for publication of the case report.

Competing interests

The authors report no conflicts of interest in this work.

\section{References}

1. Torre LA, Siegel RL, Ward EM, Jemal A. Global cancer incidence and mortality rates and trends-an update. Cancer Epidemiol Biomarkers Prev. 2016; 25(1):16-27.

2. Reck M, Rodríguez-Abreu D, Robinson AG, Hui R, Csőszi T, Fülöp $A$, et al. Pembrolizumab versus chemotherapy for PD-L1-positive non-small-cell lung cancer. N Engl J Med. 2016; 375(19): 1823- 
1833.

3. Remon J, Esteller L, Taus Á. Nivolumab plus ipilimumab combination therapy for the first-line treatment NSCLC: evidence to date. Cancer Manag Res. 2019; 11: 4893-4904.

4. Hellmann MD, Paz-Ares L, Bernabe Caro R, Zurawski B, Kim SW, Carcereny Costa E, et al. Nivolumab plus ipilimumab in advanced non-small-cell lung cancer. N Engl J Med. 2019; 381(21): 2020-2031.

5. Postow MA, Chesney J, Pavlick AC, Robert C, Grossmann K, McDermott D, et al. Nivolumab and ipilimumab versus ipilimumab in untreated melanoma. N Engl J Med. 2015;372(21):2006-2017.

6. Hammers HJ, Plimack ER, Infante JR, Rini BI, McDermott DF, Lewis LD, et al. Safety and efficacy of nivolumab in combination with ipilimumab in metastatic renal cell carcinoma: the checkmate 016 study. J Clin Oncol. 2017; 35(34): 3851-3858.

7. Gourd E. Nivolumab plus ipilimumab in metastatic colorectal cancer. Lancet Oncol. 2018; 19(3): e139.

8. Hellmann MD, Rizvi NA, Goldman JW, Gettinger SN, Borghaei H, Brahmer JR, et al. Nivolumab plus ipilimumab as recent years, first-line treatment for advanced non-small-cell lung cancer (checkmate 012): results of an open-label, phase 1, multicohort study. Lancet Oncol. 2017; 18(1): 31-41.

9. Dillard T, Yedinak CG, Alumkal J, Fleseriu M. Anti-CTLA-4 antibody therapy associated to autoimmune hypophysitis: serious immune related adverse events across a spectrum of cancer subtypes. Pituitary. 2010; 13(1):29-38.

10. Weber JS, Kahler KC, Hauschild A. Management of immune-related adverse events and kinetics of response with ipilimumab. J Clin Oncol. 2012; 30 (21): 2691-2697.

11. Kähler KC, Hassel JC, Heinzerling L, Loquai C, Mössner R, Ugurel S, et al. Management of side effects of immune checkpoint blockade by anti-CTLA-4 and anti-PD-1 antibodies in metastatic melanoma. J Dtsch Dermatol Ges. 2016; 14(7): 662-681.

12. Topalian SL, Hodi FS, Brahmer JR, Gettinger SN, Smith DC, McDermott DF, et al. Safety, activity, and immune correlates of anti-PD-1 antibody in cancer. N Engl J Med. 2012; 366(26): 2443-2454.

13. Corsello SM, Barnabei A, Marchetti P, De Vecchis L, Salvatori R, Torino F. Endocrine side effects induced by immune checkpoint inhibitors. J Clin Endocrinol Metab. 2013; 98(4): 1361-1375.

14. Faje A. Hypophysitis: evaluation and management. Clin Diabetes Endocrinol. 2016; 2(1): 15.

15. Zhou S, Khanal S, Zhang H. Risk of immune-related adverse events associated with ipilimumab-plusnivolumab and nivolumab therapy in cancer patients. Ther Clin Risk Manag. 2019; 15: 211-221.

16. Hodi FS, O'Day SJ, McDermott DF, Weber RW, Sosman JA, Haanen JB, et al. Improved survival with ipilimumab in patients with metastatic melanoma. N Engl J Med. 2010; 363(8): 711-723.

17. Torino F, Corsello SM, Salvatori R. Endocrinological side-effects of immune checkpoint inhibitors. Curr Opin Oncol. 2016; 28(4): 278-287.

18. Adrogue HJ, Madias NE. Hyponatremia. N Engl J Med. 2000; 342(21): 1581-1589.

19. Diederich S, Franzen N, Bähr V, Oelkers W. Severe hyponatremia due to hypopituitarism with adrenal insufficiency: report on 28 cases. Eur J Endocrinol. 2003; 148(6): 609-617. 
20. Iwama S, Remigis AD, Callahan MK, Slovin SF, Wolchok JD, Caturegli P. Pituitary expression of CTLA-4 mediates hypophysitis secondary to administration of CTLA-4 blocking antibody. Sci Transl Med. 2014; 6(230): 230ra45.

21. Hasegawa Y, Kawai S, Ota T, Tsukuda H, Fukuoka M. Myasthenia gravis induced by nivolumab in patients with non-small-cell lung cancer: a case report and literature review. Immunotherapy. 2017; 9(9): 701-707.

22. Hassel JC, Heinzerling L, Aberle J, Bähr O, Eigentler TK, Grimm MO, et al. Combined immune checkpoint blockade (anti-PD-1/anti-CTLA-4): evaluation and management of adverse drug reactions. Cancer Treat Rev. 2017; 57: 36-49.

\section{Figures}
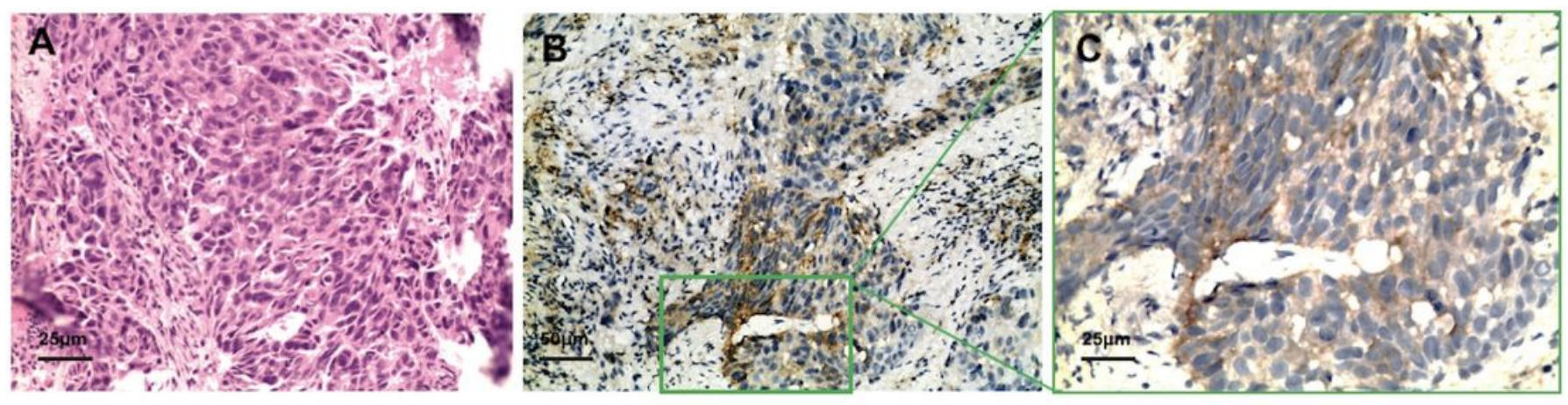

\section{Figure 1}

\section{Histopathological findings of the mass in the left upper lobe of the lung.}

A. The biopsy specimens of the mass in the lung were stained with hematoxylin and eosin. B, C. Upon immunostaining, the tumor cells were positive for membranous PD-L1 expression (magnification $\times 200$, $\times 400)$. 

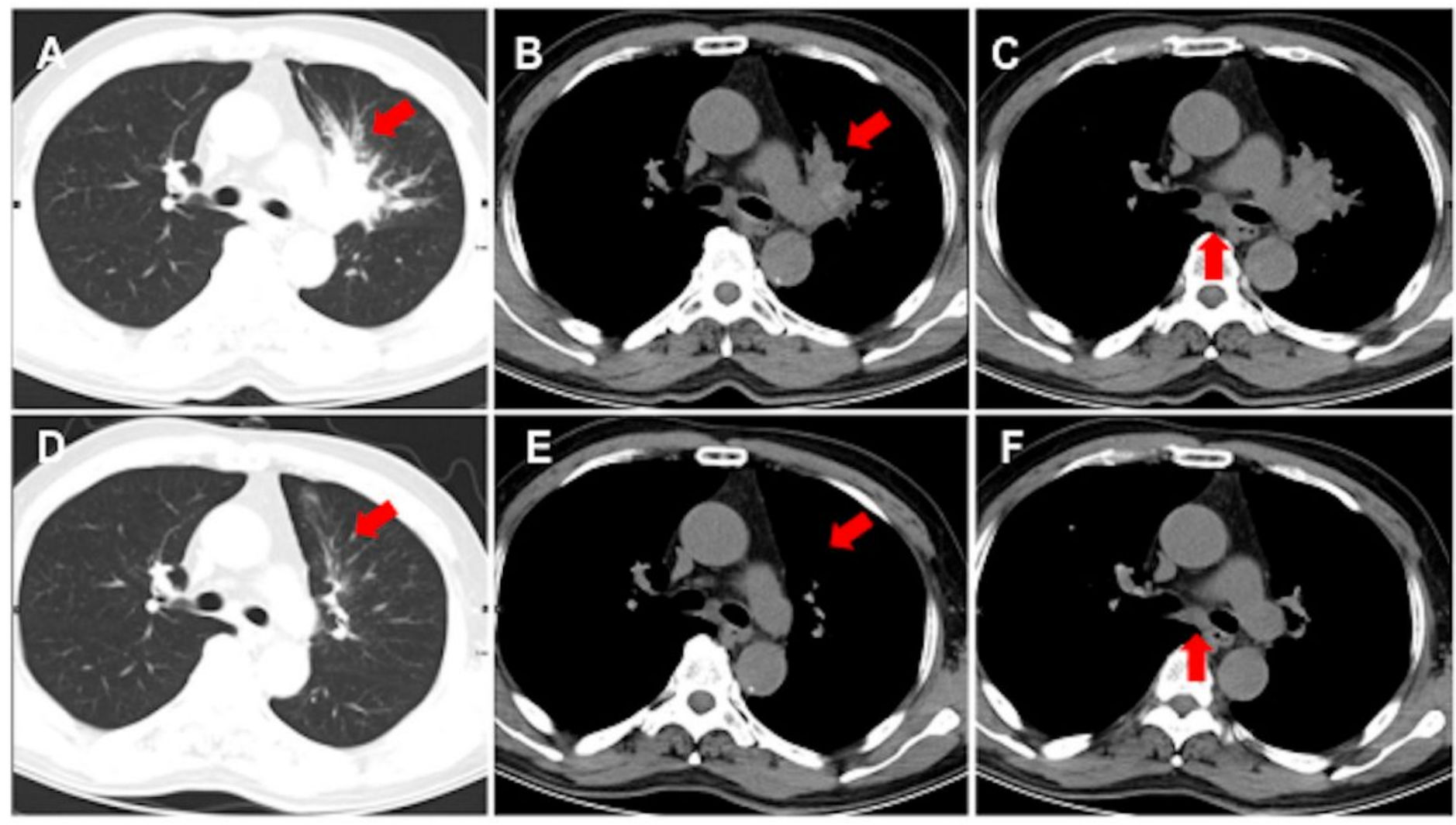

Figure 2

Images of contrastenhanced computed tomography (CT) scans.

A, B, C. Prior to the combination treatment, the primary lesion in the left upper lobe of the lung. D, E, F. After the combination treatment, the patient showed a partial response. 

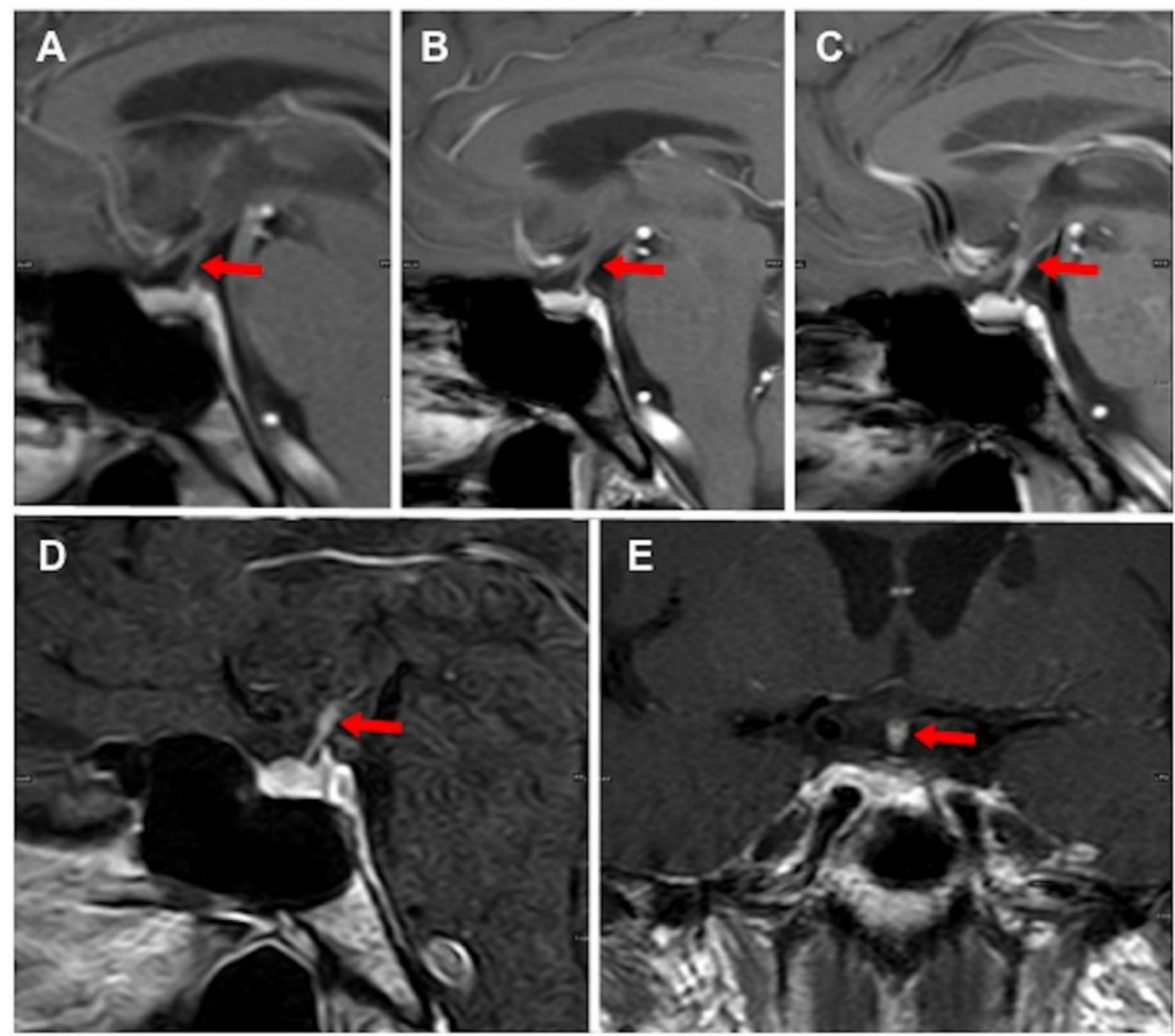

Figure 3

Images of contrastenhanced pituitary gland magnetic resonance imaging (MRI) scans.

A, B. Prior to the combination treatment. C, D, E. After the combination treatment showed obvious enhancement in pituitary stalk.
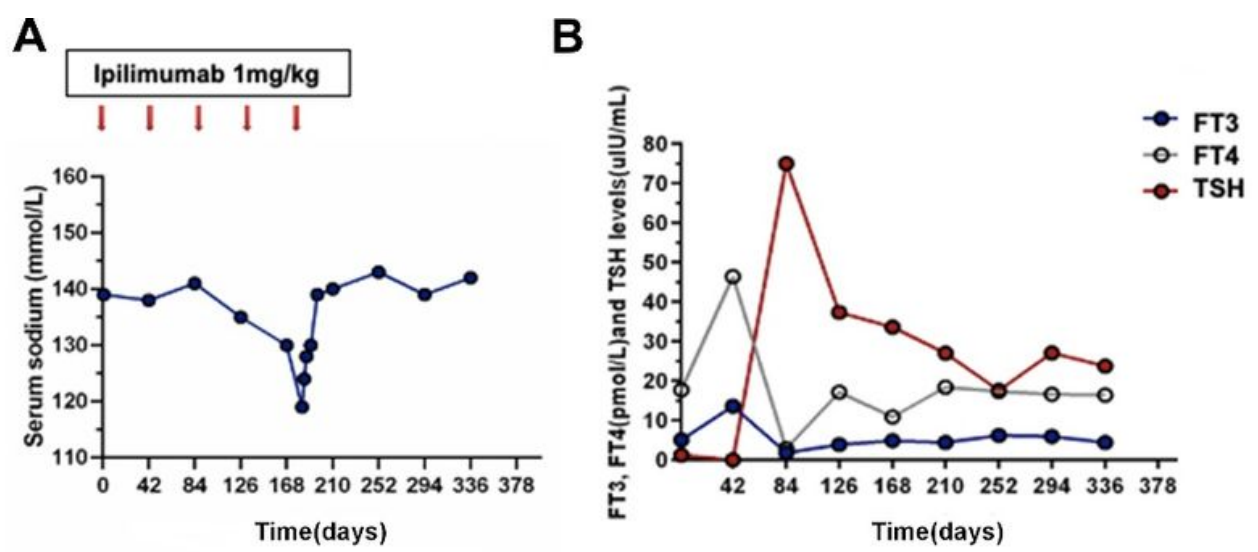

C

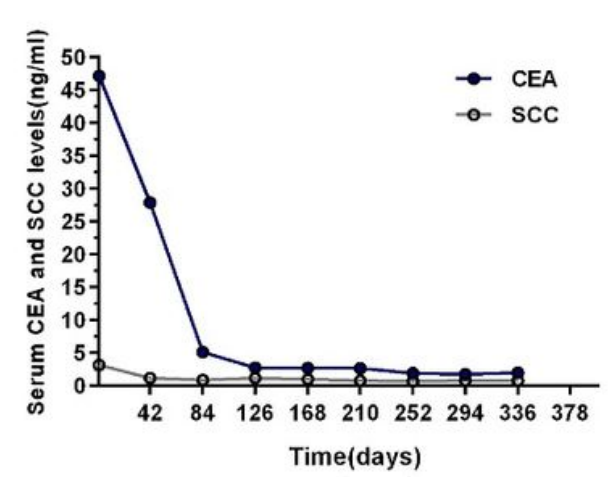

Figure 4 
Clinical course and changes in the serum sodium, thyroid hormones, CEA and SCC levels.
A. Prior to admission showing normal sodium. However, a decrease in sodium after the 13th cycle of nivolumab and the 5th cycle of ipilimumab treatment leads to severe hyponatremia. B. Consistent with serum sodium, occurrence of an increase in the serum levels of TSH and a decrease in FT4 was most likely associated with hypophysitis. C. Serum levels of CEA and SCC in the patient. Serum levels of CEA and SCC returned to normal levels after two months of treatment until the last review. 